\title{
CoMP: Efficient Monitoring for Constrained Embedded Devices
}

\author{
do Rosario, Yago Fontoura ; Fafoutis, Xenofon
}

Published in:

Proceedings of $25<$ sup $>$ th $</$ sup $>$ IEEE Symposium on Computers and Communications

Link to article, DOI:

10.1109/ISCC50000.2020.9219715

Publication date:

2020

Document Version

Peer reviewed version

Link back to DTU Orbit

Citation (APA):

do Rosario, Y. F., \& Fafoutis, X. (2020). CoMP: Efficient Monitoring for Constrained Embedded Devices. In Proceedings of 25 IEEE Symposium on Computers and Communications IEEE.

https://doi.org/10.1109/ISCC50000.2020.9219715

\section{General rights}

Copyright and moral rights for the publications made accessible in the public portal are retained by the authors and/or other copyright owners and it is a condition of accessing publications that users recognise and abide by the legal requirements associated with these rights.

- Users may download and print one copy of any publication from the public portal for the purpose of private study or research.

- You may not further distribute the material or use it for any profit-making activity or commercial gain

- You may freely distribute the URL identifying the publication in the public portal

If you believe that this document breaches copyright please contact us providing details, and we will remove access to the work immediately and investigate your claim 


\title{
CoMP: Efficient Monitoring for Constrained Embedded Devices
}

\author{
Yago Fontoura do Rosário and Xenofon Fafoutis \\ Technical University of Denmark, Denmark \\ Email: yago.rosario@hotmail.com.br, xefa@dtu.dk
}

\begin{abstract}
Network monitoring has always being a challenge for network administrators. Their tasks are to keep track of every node in a network and ensure that they behave correctly. The Simple Network Management Protocol (SNMP) was designed to aid network administrators in their everyday tasks. A new wave of devices has been coming in the last years where small embedded devices are also being connected to the network. These new devices are part of the Internet of Things (IoT). IoT devices differ in many aspects from traditional networks. However, the main challenge with IoT devices is their hardware constraints (very limited memory and processing resources) and their limited energy budget. Indeed, they are usually powered by batteries and can be deployed in harsh environments. The current monitoring protocols were designed without taking the constraints of IoT devices into account. In an IoT context, there are very limited resources available for monitor constrained devices. While the current protocols do work with these devices they have an unnecessary overhead resulting in a waste of resources that could otherwise be used for some other task, or to save energy. This work proposes a new protocol designed for embedded devices and evaluates it against the state of the art.
\end{abstract}

Index Terms-SNMP, CoAP, IoT, Internet of Things, Sensor, WSN, Wireless Sensor Networks, Monitoring

\section{INTRODUCTION}

The emerging Internet of Things (IoT) finds billion connected devices in every aspect of society. Managing this amount of devices is not an easy task. Other than their sheer volume, they are also heavily resource-constrained which makes everything more complicated. Although not every device has to be monitored, there will be key devices that cannot fail. Consider a train scheduling and signaling system that relies heavily on a Wireless Sensor Network (WSN) due to the low operational cost over a conventional wired network. Yet, the trade-off is the additional network administrators that have to be employed to monitor the behavior and correct operation of such a critical network. A simple failure in this network can cause anything from a simple delay to a severe train crash.

The current monitoring protocols were designed without taking the constraints of WSN nodes into account. One does not want to use many resources to monitor constrained devices. While the current protocols do work with these devices they have an unnecessary overhead. This paper analyzes and evaluates the current solutions for network monitoring in a WSN context. Our base hypothesis is that the current technologies use too many resources and they are too heavy for constrained devices. Such an assumption stems from the fact that the standard protocol for network monitoring (i.e. Simple Network Management Protocol, SNMP) was developed in the 1980s.

In this paper we introduce CoMP (Constrained Monitoring Protocol). CoMP provides the same functionality as the current state-of-the-art protocols, yet in a much more resourceefficient manner. We have implemented CoMP for the Contiki$\mathrm{NG}^{1}$ operating system and compared it against monitoring standards, including SNMP [1] and CoAP (Constrained Application Protocol) [2]. Specifically, we compare the protocols in terms of (i) code footprint, (ii) application-layer overhead, (iii) over-the-air bytes, and (iv) radio energy usage.

The rest of this paper is structured as follows. Section II presents some related work whereby the issue of monitoring WSNs was addressed. Section III presents our proposed monitoring protocol CoMP that is designed to work better in a WSN context. Section IV provides experiments that evaluate and compare CoMP against SNMP and CoAP. Finally, Section V concludes the paper.

\section{RELATED WORK}

Since it is clear that there has not been defined a standard way of monitoring resources on embedded systems, several papers have proposed either new protocols or adaptation of the current protocols to have a good way to perform this action.

On [3] a new protocol for monitoring IoT devices is proposed. The LoWPAN Network Management Protocol (LNMP) targets two problems faced in these networks, Network Discovery and Device Monitoring. Since the deployment is usually done in an ad hoc fashion, a normal problem when monitoring IoT devices is to keep track of which nodes are present on the network. This could be done manually, but in a very dense network, this would be a massive task and very prone to errors. To target this issue, a Network Discovery proposal was done. In this, the end devices report to a coordinator their status periodically. This is done recursively until it reaches the gateway. During normal operations only the changes on the state table are reported upwards, reducing the traffic. The gateway likewise has a state table, like any other device in the network. However, its table is available in a Management Information Base (MIB), this way management systems can be aware of the devices available in the network. To target the resource monitoring problem an adaptation layer is proposed. A conversion between the SNMP Object Identifier

\footnotetext{
${ }^{1}$ www.contiki-ng.org
} 
(OID) to a 6LoWPAN OID is the only modification proposed. Another proposed improvement is caching in the gateway where if a value is constant for the entire network the gateway replies instantly without sending the request to the end device. The Network Discovery solution is really solid since it targets a real issue in dense networks, however, the only compression proposed in the SNMP was a conversion between OIDs which is good, but not enough. In CoMP, the OID system is kept but compression is applied to it. However, it does not address the Network Discovery problem because this can be solved using the Physical Topology MIB proposed on [4]. This MIB was designed to be used with the Link Layer Discovery Protocol (LLDP) which was proposed on [5]. But this can also be filled in with the RPL Neighbors Table information.

On [6], the SNMP for 6LoWPAN (6LoWPAN-SNMP) was proposed. The same principle behind the 6LoWPAN was used. The SNMP headers were analyzed and compressed as much as possible. Some fields are way too large, only a small fraction of it is usually used. For example, the header version field which is a 4-byte integer can easily be compressed into 3bit since he proposes 4 new versions. This limits the number of new versions since all 3 bits are used. Other fields like the Request ID can be limited too, there is no need to use 4 bytes in total for this. The main reason for this identification is to avoid duplicate messages, but it is almost impossible to have 4294967296 messages being handled at the same time, especially in a WSN context. For this reason, it proposed to limit it to 255 which only requires 1 byte. No details on the encoding were given, so it is possible to assume that the same BER (Basic Encoding Rules) encoding was used. The OID system was kept and no compression was suggested for it. Lastly, the same proxy technique as before was used in the border gateway to convert the messages from SNMP to 6LoWPAN-SNMP and vice versa. CoMP uses a different encoding which targets both key requirements for constrained devices, namely the code footprint and and the encoded buffer size. Also, an OID compression technique is used.

On [7], the EmNetS Network Management Protocol (EMP) is proposed. Just like the LNMP, it tackles two problems of the WSN, Network Discovery and Monitoring. To solve the first, it uses a similar approach. There are Coordinators and End Devices, the coordinators are responsible for keeping track of its end devices. This is done recursively until the Gateway is reached. On the gateway a table with all the devices and the last time its entry was updated are kept, this can be used to create several statistics. To solve the second problem and keep this solution SNMP compliant, a new MIB structure was proposed that will be maintained only for WSN devices. Currently, the MIB scope is huge, meaning that many of those entries are either deprecated, not used or will never be used in the WSN context. This way only the MIBs that are used in this context will be considered, reducing the depth of the tree. In the gateway, a conversion table is available where the OIDs can be converted into an equivalent EMP OIDs. Another trick used to reduce the bandwidth is to keep all constant values in a cache on the border gateway. This way the gateway can respond to the request without forwarding this request into the WSN. A good example of which variable is always constant in a system until it is updated is the system description (sysDescr). In this work, the Network Discovery problem is not tackled, but to solve the Monitoring problem better techniques to reduce the Network usage are used and the OID is kept, no new MIBs are introduced.

These approaches tried to follow the same principle used by the 6LoWPAN when an adaptation layer was proposed. This ensures the interoperability between these protocols. This approach ensures seamless integration of WSNs into existing ecosystems. It is fairly simple to use the same monitoring system, like PRTQ 2 , that currently monitors all networks of a ISP to additionally monitor the WSN that this company has. In this work a new protocol is proposed, CoMP, which is a standalone protocol, like CoAP.

\section{Constrained Monitoring Protocol (CoMP)}

In this section, we introduce CoMP. Its goal is to be a very lightweight monitoring protocol to be used in a WSN context. Additionally, it has the objective to be SNMP compliant, therefore it should be possible to convert SNMP requests into CoMP requests. However, in this paper, the focus area is the monitoring requests from SNMP (i.e. Get, Get-Next, Get-Bulk, and Response). The Set, Trap and Inform requests are not covered but the proposed structure should cover these requests out-of-the-box.

In this regard, CoMP offers resource-efficient equivalents to SNMPv1 and SNMPv2 [8], which are referred to as CoMPv1 and CoMPv2 respectively.

\section{A. Packet Structure}

The CoMP packet is defined by a header, an extra header, and a PDU (Protocol Data Unit). The header is shared by CoMPv1 and CoMPv2, shown in Figure 1 It is pretty simple, the first information in it is the protocol version which is an unsigned 4-bit integer and it is followed by the operation which is too an unsigned 4-bit integer.

\begin{tabular}{|l|l|}
\hline 0 & \multicolumn{1}{|c}{4} \\
\hline Version & Operation \\
\hline
\end{tabular}

Fig. 1. CoMPv1 and CoMPv2 header structure.

If the operation is a Get or Get-Next the extra header will look like in Figure 2, which has a Request ID (unsigned 8-bit integer), followed by the Error Code (unsigned 5-bit integer), and lastly the Error Index (unsigned 3-bit integer).

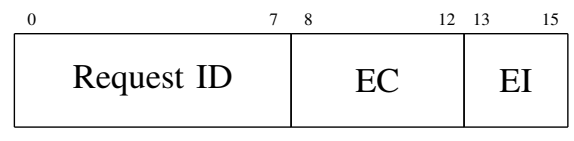

Fig. 2. CoMPv1 and CoMPv2, Get and Get-Next extra header.

\footnotetext{
${ }^{2}$ https://www.paessler.com/prtg
} 
However, if the version is CoMPv2 and the operation is the Get-Bulk a different extra header is used (Figure 3). The first element is a Request ID (unsigned 8-bit integer), yet it is followed by the Non-Repeaters (unsigned 4-bit integer), and finally the Max Repeaters (unsigned 4-bit integer).

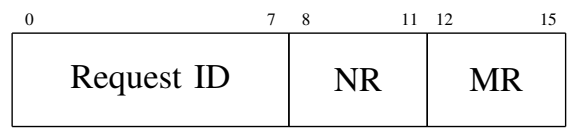

Fig. 3. CoMPv2, Get-Bulk extra header.

Depending on the operation the CoMP PDU carries different information. In any request, Get, Get-Next or Get-Bulk, the PDU is a list of OIDs that the client is requesting the server, as shown in Figure 4

\begin{tabular}{|c|}
\hline OID 1 \\
\hline \hline OID 2 \\
\hline \hline$\cdots$ \\
\hline \hline OID $\mathrm{n}$ \\
\hline
\end{tabular}

Fig. 4. CoMP PDU variables on request packet.

If the CoMP operation is a Get, the response is a list of Values, as shown in Figure 5. This list is indexed using the same position as the OID in the request. For example, if the client requests the list [1.3.6.1.2.1.1.1.0,1.3.6.1.2.1.1.3.0], the server would reply ["sysDescr", sysUptime].

\begin{tabular}{|c|}
\hline Value 1 \\
\hline \hline Value 2 \\
\hline \hline$\ldots$ \\
\hline \hline Value $\mathrm{n}$ \\
\hline
\end{tabular}

Fig. 5. CoMP PDU values on response packet.

However, the same method to reduce the message size cannot be used in the Get-Next or Get-Bulk operation, since the server replies with the first OID after the requested one. The next OID is unknown to the client, so the server has to reply with the OID and the corresponding value. For this, a simple array is used where the first element is the OID and the second is the value, as shown in Figure 6.

\section{B. Encoding}

Following the CoAP's encoding proposal. All the CoMP headers are binary encoded and using the network byte order,

\begin{tabular}{|c|}
\hline [OID 1, Value 1] \\
\hline \hline$[$ OID 2, Value 2] \\
\hline$\ldots$ \\
\hline \hline [OID n, Value n] \\
\hline
\end{tabular}

Fig. 6. CoMP PDU variable names and values on response packet.

however in the current proposal there is no 16-bit or higher integer, therefore, the byte order is irrelevant. All the PDU variables, values or the pair variable and value, were encoded using the CBOR (Concise Binary Object Representation) encoding. Proposed on [9] in 2013, it targets constrained devices since one of its goals is to allow a small code footprint, very small messages and to allow extensions without the need for new versions.

The CBOR encoding has a data item header that contains a major type and additional information. For each major type, the additional information contains different information that can be used to process the rest of the data. In Figure 7 Figure 8 , and Figure 9 the three data item field encodings are shown.

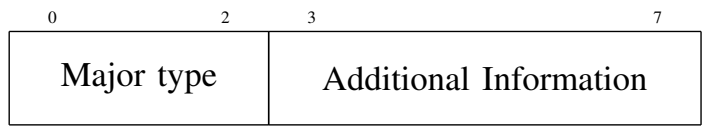

Fig. 7. CBOR tiny field encoding.

\begin{tabular}{|c|c|}
\hline 0 & 3 \\
\hline Major type & Additional Information \\
\hline & Value \\
\hline \multicolumn{2}{|c|}{$\ldots \times$ Byte Count ... } \\
\hline
\end{tabular}

Fig. 8. CBOR short field encoding.

\section{Messaging Pattern}

Similarly to SNMP and CoAP, CoMP supports the Request-Response message pattern.

\section{Resource Identification}

Since the CoMP is designed to be SNMP compliant, it employs the same resource identification technique. Even though this constitutes protocol overhead, the OID is very good for large networks, but this implies a huge depth in the OID size and requires a centralized institution to manages this. Since the centralized institution never became a problem in all these years and the OIDs can be compressed, it is feasible to use this mechanism in in WSN context. 


\begin{tabular}{|c|c|}
\hline Major type & Additional Information \\
\hline \hline & Payload length \\
\hline$\ldots \times$ Byte Count $\ldots$ \\
\hline Value \\
\hline$\ldots \times$ Payload length $\ldots$ \\
\hline
\end{tabular}

Fig. 9. CBOR long field encoding.
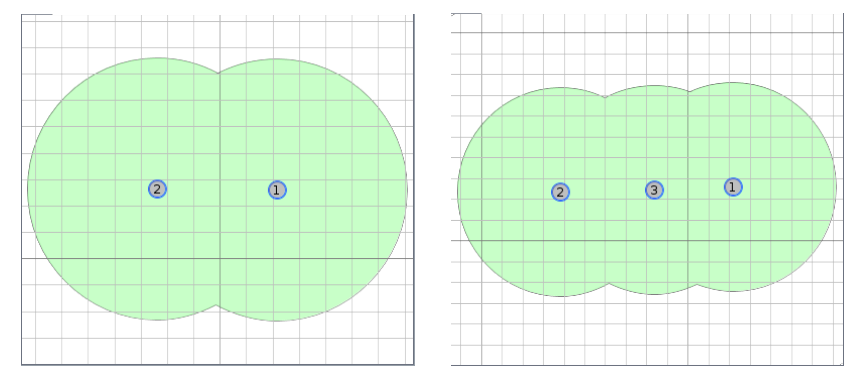

(a) Topology 1: One server (2) and one border router (1).

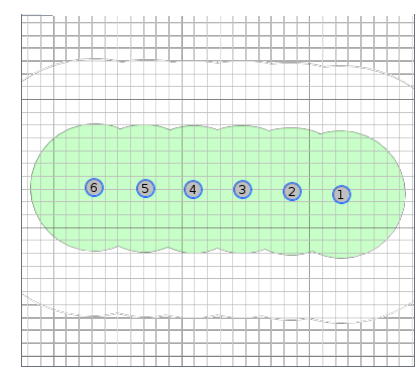

(b) Topology 2: Two servers $(2,3)$ and one border router (1).

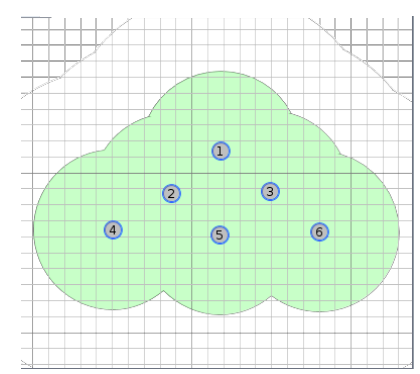

(c) Topology 3: Five servers (2-6) (d) Topology 4: Five servers (2-6) and one border router (1) in a line and one border router (1) in a tree topology.

Fig. 10. Topologies simulated in Cooja.

\section{E. Monitored Values}

The monitored values are organized the same way as the SNMP, again to be compliant. Therefore the MIB structure is used. Even though MIBs are defined using the ASN.1 specification this does not affect the protocol itself. This is only a standardized way to write MIBs and share them between systems or from a manufacturer to a client.

\section{Evaluation}

\section{A. Experiments}

We conduct three experiments to evaluate CoMP and compare it against SNMP and CoAP. The experiments are based on a proof-of-concept implementation of CoMP for Contiki$\mathrm{NG}^{3}$. For the purposes of this work, we also developed

\footnotetext{
$\sqrt[3]{\text { https://github.com/Yagoor/contiki-ng/tree/comp }}$
}

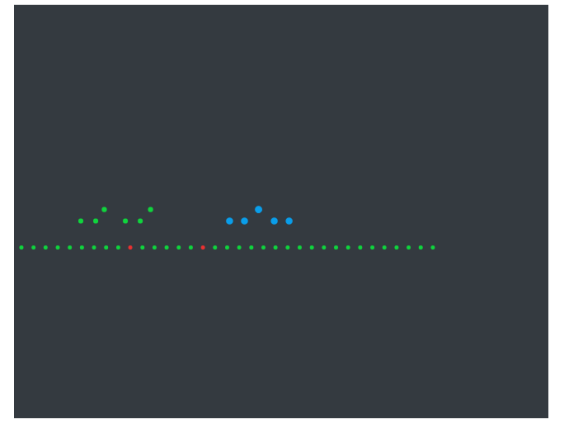

Fig. 11. FIT IoT-LAB Topology: four servers (Blue dots in line) and one border router (Blue dot above Blue line). The DODAG was built with a directly link from the border router to the servers

TABLE I

CODE FOOTPRINT ON CC26X0-CC13X0 [BYTES]

\begin{tabular}{|c|c|c|c|}
\hline & RAM & ROM & Total \\
\hline SNMP & $1120(+1.82 \%)$ & $3140(-2.67 \%)$ & $4260(-1.29 \%)$ \\
\hline CoAP & $1986(+80.55 \%)$ & $9724(+202 \%)$ & $11710(+173 \%)$ \\
\hline CoMP & 1100 & 3216 & 4316 \\
\hline
\end{tabular}

and contributed to the Contiki-NG source code a standardcompliant implementation of SNMPv1 and SNMPv2 4

SNMP and CoMP have a well defined way to store the monitored values and a defined resource identification method. However, CoAP is more generic. In order to make it suitable for this experiment a standardized way of mapping CoAP resources to monitored values was defined. To use the CoAP as a monitoring protocol the approach was to have the path to have the same SNMP MIB and to use a query named info with the value of the element, that was the same name used in the SNMP. If no query is sent to the endpoint a list with all the available monitored values are sent as a response, this method can be used to act similar to the SNMP Walk. Since the key argumentation to use CoAP as a monitoring protocol is because it is human-friendly, a very descriptive method was utilized and the JSON encoding was used.

The first experiment evaluates the code footprint (ROM and RAM) of each protocol. To this end, a compilation of a Contiki-NG server example for the $\mathrm{cc} 26 \times 0-\mathrm{cc} 13 \times 0$ target was done. The goal is to see how much memory resources a module uses on a typical IoT device.

The second series of experiments assesses the network usage using the Cooja Simulator [10]. The Cooja simulations were used to analyze and evaluate many 6LoWPAN protocols like it was done for RPL [11] and for CoAP [12]. Figure 10] shows the four topologies that we considered. For the remainder of this section we refer to these four topologies as Topology 1-4 respectively. We evaluate the network usage in these scenarios for various reasons. The first one is the resource limitation, the least data is transferred the better. Moreover, the physical service data unit is limited to 127 bytes therefore, it is a good idea to have a protocol that fits inside of it, avoiding

\footnotetext{
${ }^{4}$ https://github.com/contiki-ng/contiki-ng/pull/1020
} 


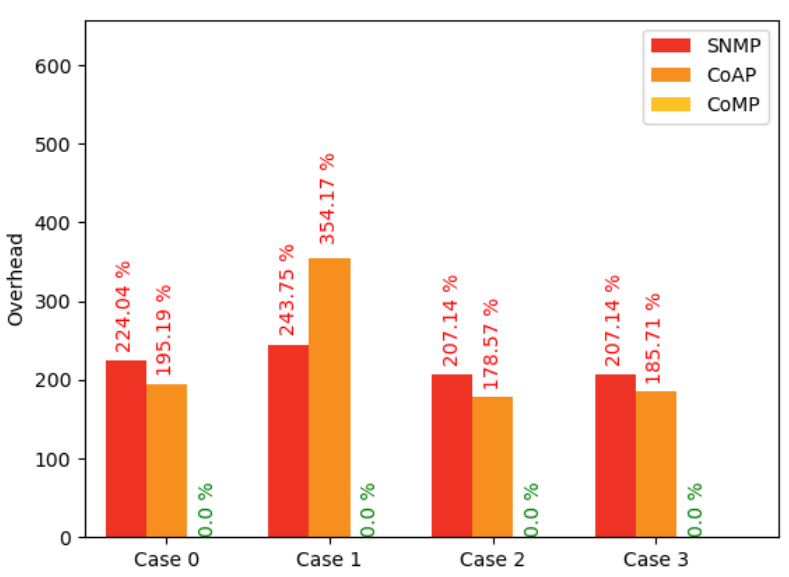

Fig. 12. Relative application-layer overhead of request packets relative to CoMP.

TABLE II

APPliCATION LAYER NETWORK USAGE [Bytes]

\begin{tabular}{|c|c|c|c|c|c|c|c|c|}
\hline & \multicolumn{2}{|c|}{ Case 0 } & \multicolumn{2}{c|}{ Case 1 } & \multicolumn{2}{c|}{ Case 2 } & \multicolumn{2}{c|}{ Case 3 } \\
\cline { 2 - 9 } & RES & REQ & RES & REQ & RES & REQ & RES & REQ \\
\hline SNMP & 468 & 337 & 352 & 165 & 85 & 43 & 45 & 43 \\
\hline CoAP & 407 & 307 & 371 & 218 & 68 & 39 & 29 & 40 \\
\hline CoMP & 258 & 104 & 216 & 48 & 48 & 14 & 7 & 14 \\
\hline
\end{tabular}

the expensive fragmentation. Hence the key value that has to be analyzed is the transferred data size. Anything under the application layer is out of the protocol's control because all the protocols used in this project are within this layer but it is furthermore necessary to analyze the bytes over the air because this determines the energy usage.

In each topology, four use cases are studied (Case 0-3). Every node, except the border router, is requested by a client outside the WSN. Case 0 is a Walk where all the monitored values available on the server are requested one by one. Case 1 is a Bulk Walk where all the monitored values are requests, two at a time. Only two were used because this increases the packet size and a balance has to be found between these two attributes. Additionally, in Case 2, a system description is asked, in this case, it is the operating system name and version tag. Lastly in Case 3, the system uptime is asked. The two last cases are used to analyze how much data each protocol uses to transmit a string and an integer.

The third experiment evaluates the radio activity time of each protocol in a test-bed environment. For these experiments, the TSCH (Time-Slotted, Channel Hopping) protocol is used because it has a good overall performance in terms of energy and reliability [13], [14]. The scheduler used is the Orchestra [15]. The RPL's and TSCH's probing interval was tuned using the five nines reliability proposed on [16], which reduces the impact of the beacons on the experiments. The experiment was conducted on the FIT IoT-LAB [17] located in Grenoble, France. This laboratory is composed of several microcontrollers of different architectures. In this paper, the M3 nodes were used since they represent the state-of-the-

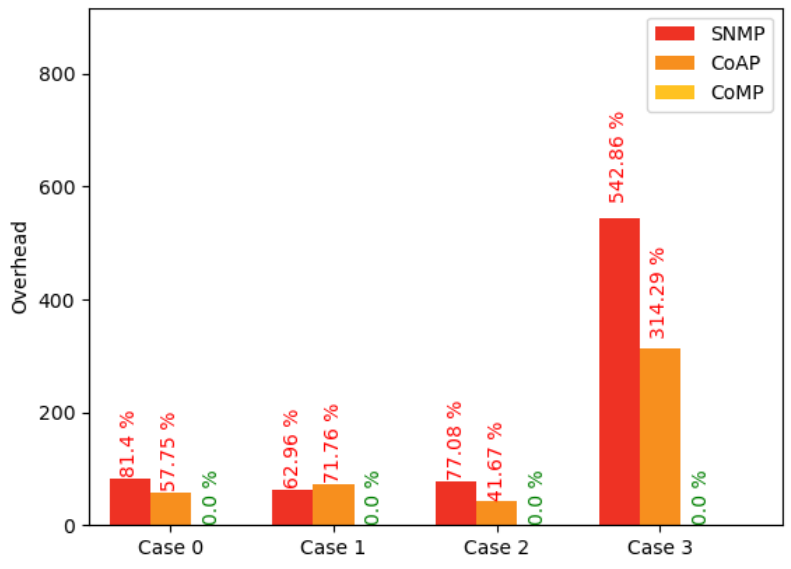

Fig. 13. Relative application-layer overhead of response packets relative to CoMP.

art WSN devices. This testbed was used in several papers to evaluate protocols in real-life scenarios like on [18] and [19]. To estimate the radio activity time the Energest Module was used which is a software-based online mechanism for energy estimation. It simply counts the number of ticks spent in different states. Energest has been used to track the energy consumption of IoT devices in real-world deployments [20]. In this evaluation, we focus on the radio duty cycle (Radio TX and Radio RX states).

The goal of this experiment is to prove that by reducing the bytes over the air in a protocol will impact positively the energy consumption which is still the biggest constraint in the WSN environment. The topology used in this experiment can be seen in Figure 11. In these tests it is mostly interesting to look into the Radio Activity Time during transmission (TX) because the receiving (RX) is controlled by the scheduler of the MAC protocol, therefore the only radio activity time where the application-layer protocol can influence positively or negatively is the TX cycle. Additionally, the DODAG was built in a way that the border router has a direct route to every server, which in this scenario is good because to analyze the radio activity time of an application layer protocol is necessary to run the same experiment multiple times and get the smallest value generated by it. This is necessary because a synchronization beacon from the MAC protocol can be sent during the execution of the protocol and it will taint the evaluation. Another unwanted behavior is retransmission that can be caused by some external interference like a packet collision. In this experiment the medium is totally out of control and collisions are likely to happen, this would too affect the evaluation. Instead of running the same experiment multiple times to get the smallest value, which is the one with the least probability of external interference, it is possible to get the smallest activity time from the four nodes either in transmission and reception. Therefore in this experiment, every node, except the border router, is requested by a client outside the WSN. 
TABLE III

BYTES OVER THE AIR IN TOPOLOGY 1

\begin{tabular}{|c|c|c|c|c|c|c|c|c|}
\hline & \multicolumn{2}{|c|}{ Case 0 } & \multicolumn{2}{c|}{ Case 1 } & \multicolumn{2}{c|}{ Case 2 } & \multicolumn{2}{c|}{ Case 3 } \\
\cline { 2 - 9 } & RES & REQ & RES & REQ & RES & REQ & RES & REQ \\
\hline SNMP & 874 & 761 & 562 & 377 & 141 & 96 & 94 & 96 \\
\hline CoAP & 813 & 731 & 637 & 483 & 117 & 92 & 78 & 93 \\
\hline CoMP & 650 & 528 & 412 & 260 & 97 & 67 & 56 & 67 \\
\hline
\end{tabular}

TABLE IV

BYTES OVER THE AIR BYTES IN TOPOLOGY 2

\begin{tabular}{|c|c|c|c|c|c|c|c|c|}
\hline \multirow{2}{*}{} & \multicolumn{2}{|c|}{ Case 0 } & \multicolumn{2}{c|}{ Case 1 } & \multicolumn{2}{c|}{ Case 2 } & \multicolumn{2}{c|}{ Case 3 } \\
\cline { 2 - 9 } & RES & REQ & RES & REQ & RES & REQ & RES & REQ \\
\hline SNMP & 2676 & 2411 & 1702 & 1195 & 423 & 304 & 291 & 304 \\
\hline CoAP & 2499 & 2321 & 1935 & 1529 & 360 & 292 & 246 & 295 \\
\hline CoMP & 2022 & 1712 & 1268 & 844 & 300 & 217 & 177 & 217 \\
\hline
\end{tabular}

\section{B. Results}

Table I summarizes the code footprint information of the three protocols. It is evident that CoAP performs the worst, whilst SNMP has a slightly better performance than CoMP on ROM usage. CoMP is slightly better than SNMP on RAM usage. The table also shows the overhead relative to CoMP.

Next, we present the results of the Cooja simulations. Table II summarizes the application-layer network usage information of the three protocols in all four cases. In particular, the table reports the total number of application-layer bytes transmitted in requests packets (REQ) and response packets (RES) respectively. It is clear that CoMP offers a significant improvement. Figure 12 and Figure 13 present the relative overhead of the request and response packets with respect to CoMP for each case. In all cases, CoMP performed much better than the other protocols in all cases.

Table III shows the total bytes transmitted over the air in request (REQ) and response (RES) packets respectively for Topology 1. Similarly, Table IV] Table V and Table VI summarize the total bytes over the air for Topology 2, Topology 3 and Topology 4 respectively. In all topologies and cases, CoMP outperforms the other protocols.

The biggest downside of CoAP is in Case 0 and 1 where it is first necessary to request a list of all monitored values that are available before starting the walk request. This disadvantage cannot be seen in Case 0 because on a traditional walk the client requests the next value after the one he is sending, therefore it does not know beforehand which is the last one available. It keeps asking until it is replied with the End of View message. In CoAP it asks for all monitored values available, so it knows the last one beforehand. The number of requests is the same because both have an extra request. But in Case 1, the SNMP sends the End of View message together with the last value eliminating the extra request.

Finally, we summarize the results of the test-bed experiments in the FIT/IoT Lab in Table VII In particular, the table shows the total radio activity time (in ms) for transmitting request and response packets respectively. Similarly, Figure 14 and Figure 15 illustrate the relative overhead of transmitting request and response packets with respect to the best per-
TABLE V

BYTES OVER THE AIR BYTES IN TOPOLOGY 3

\begin{tabular}{|c|c|c|c|c|c|c|c|c|}
\hline \multirow{2}{*}{} & \multicolumn{2}{|c|}{ Case 0 } & \multicolumn{2}{c|}{ Case 1 } & \multicolumn{2}{c|}{ Case 2 } & \multicolumn{2}{c|}{ Case 3 } \\
\cline { 2 - 9 } & RES & REQ & RES & REQ & RES & REQ & RES & REQ \\
\hline SNMP & 13650 & 14080 & 8590 & 6980 & 2115 & 1775 & 1500 & 1775 \\
\hline CoAP & 12765 & 13525 & 9765 & 8914 & 1845 & 1700 & 1275 & 1715 \\
\hline CoMP & 10470 & 10480 & 6500 & 5180 & 1545 & 1325 & 930 & 1325 \\
\hline
\end{tabular}

TABLE VI

BYTES OVER THE AIR IN TOPOLOGY 4

\begin{tabular}{|c|c|c|c|c|c|c|c|c|}
\hline & \multicolumn{2}{|c|}{ Case 0 } & \multicolumn{2}{c|}{ Case 1 } & \multicolumn{2}{c|}{ Case 2 } & \multicolumn{2}{c|}{ Case 3 } \\
\cline { 2 - 9 } & RES & REQ & RES & REQ & RES & REQ & RES & REQ \\
\hline SNMP & 7154 & 6472 & 4544 & 3208 & 1128 & 816 & 779 & 816 \\
\hline CoAP & 6666 & 6232 & 5158 & 4104 & 963 & 784 & 659 & 792 \\
\hline CoMP & 5416 & 4608 & 3392 & 2272 & 803 & 584 & 475 & 584 \\
\hline
\end{tabular}

forming protocol respectively. In all cases, CoMP consumes less energy than the other protocols. More specifically, SNMP slightly outperforms CoMP in the response packets of Case 0 and Case 3, yet the improvements in the request packets are much larger, making CoMP more energy-efficient in total. As mentioned before, the receiving (RX) cycle is controlled by the scheduler and the network is prone to external interference which may cause a different number of retransmissions in each case. Therefore, the small difference between SNMP and CoMP in the response packets are due to the MAC protocol and not because the application-layer protocol is better.

\section{CONClusion}

This paper analyzes SNMP and CoAP, and proposes a new protocol that offers the same functionality as the former whilst having a constrained design like the latter.

SNMP proved to be extremely structured when used for network monitoring, hence why it is still the most used protocol for this purpose since the 1980s. It also has a small code footprint due to its simplicity. However, the amount of bytes transmitted in the application layer is massive, due to its encoding technique and because the request is like a questionnaire that the server fills in with the answers.

CoAP is very human-friendly in its design mainly because it was designed to be the HTTP for constrained devices. It has a very small header which considerably reduces the number of bytes in the application layer but it uses the URI for identification which means strings and consequentially a significant amount of bytes for resource identification. Additionally, it implements all the REST functionalities which are not necessary for network monitoring since the only method necessary would be the Get. This increases the code footprint significantly.

Instead, CoMP was designed to have the best of both worlds. It incorporates the messaging pattern, Request-Response, resource identification, OID, monitored values, MIB, and operations, Get, Get-Next and Get-Bulk, from SNMP. It uses the binary encoding for the header and the CBOR encoding for the body just like it can be done with CoAP. This design proved to have a really good code footprint, equivalent to 


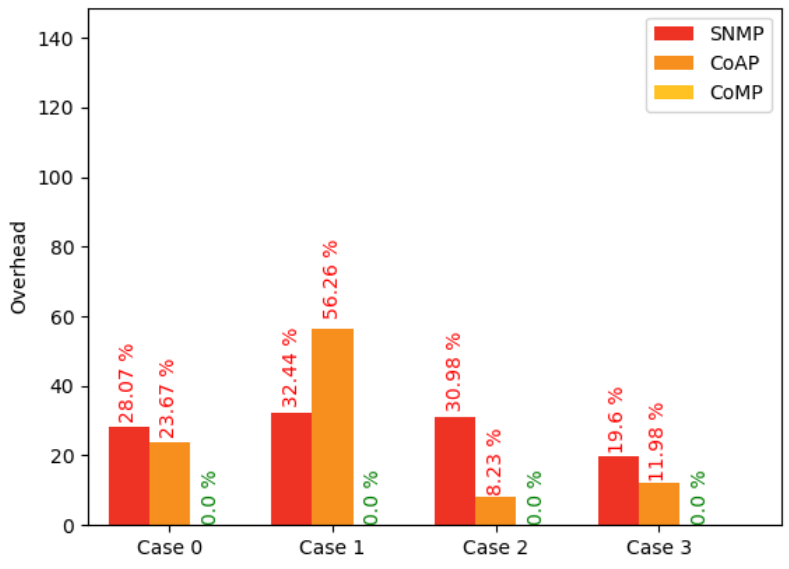

Fig. 14. Relative radio activity time (ms) for transmitting request packets with respect to CoMP in FIT/IoT Lab test-bed experiments.

TABLE VII

RADIO ACTIVITY TIME IN FIT/IOT LAB [MS

\begin{tabular}{|c|c|c|c|c|c|c|c|c|}
\hline & \multicolumn{2}{|c|}{ Case 0 } & \multicolumn{2}{c|}{ Case 1 } & \multicolumn{2}{c|}{ Case 2 } & \multicolumn{2}{c|}{ Case 3 } \\
\cline { 2 - 9 } & RES & REQ & RES & REQ & RES & REQ & RES & REQ \\
\hline SNMP & 56,79 & 41,93 & 34,17 & 27,80 & 11,65 & 10,19 & 10,55 & 7,69 \\
\hline CoAP & 61,49 & 40,49 & 43,51 & 32,80 & 13,82 & 8,42 & 14,00 & 7,20 \\
\hline CoMP & 58,83 & 32,74 & 32,98 & 20,99 & 10,77 & 7,78 & 10,77 & 6,43 \\
\hline
\end{tabular}

SNMP. The difference was minimum and it was in favor of SNMP because CoMP implements an OID compression which increases the code footprint but it reduces the bytes transmitted when multiple OIDs are present. The bytes transmitted in the application layer were reduced significantly, up to $85 \%$ in some cases. This reduction reflected in the bytes over the air which, in turn, affects the radio duty cycle that is directly tied to energy consumption.

In future work, we plan to add a security layer in CoMP, adopting elements of SNMPv3 [21].

\section{REFERENCES}

[1] J. Case, M. Fedor, M. L. Schoffstall, and J. Davin, "RFC1157: Simple network management protocol (snmp)," 1990.

[2] Z. Shelby, K. Hartke, C. Bormann, and B. Frank, "Constrained application protocol (coap), draft-ietf-core-coap-13," Orlando: The Internet Engineering Task Force-IETF, 2012.

[3] H. Mukhtar, K. Kang-Myo, S. A. Chaudhry, A. H. Akbar, K. Ki-Hyung, and Seung-Wha Yoo, "LNMP- Management architecture for IPv6 based low-power wireless Personal Area Networks (6LoWPAN)," in NOMS 2008 - 2008 IEEE Network Operations and Management Symposium, 042008 , pp. 417-424.

[4] A. Bierman and K. Jones, "Physical Topology MIB," Internet Requests for Comments, RFC Editor, RFC 2922, 09 2000. [Online]. Available: http://www.rfc-editor.org/rfc/rfc2922.txt

[5] I. C. Society, "IEEE Standard for Local and metropolitan area networks - Station and Media Access Control Connectivity Discovery," IEEE Std 802.1AB-2005, pp. 1-176, 052005.

[6] H. Choi, N. Kim, and H. Cha, "6LoWPAN-SNMP: Simple Network Management Protocol for 6LoWPAN," in 2009 11th IEEE International Conference on High Performance Computing and Communications, 06 2009, pp. 305-313.

[7] S. A. Chaudhry, G. Boyle, W. Song, and C. Sreenan, "EMP: A Network Management Protocol for IP-based Wireless Sensor Networks," in 2010 International Conference on Wireless and Ubiquitous Systems, 10 2010, pp. 1-6.

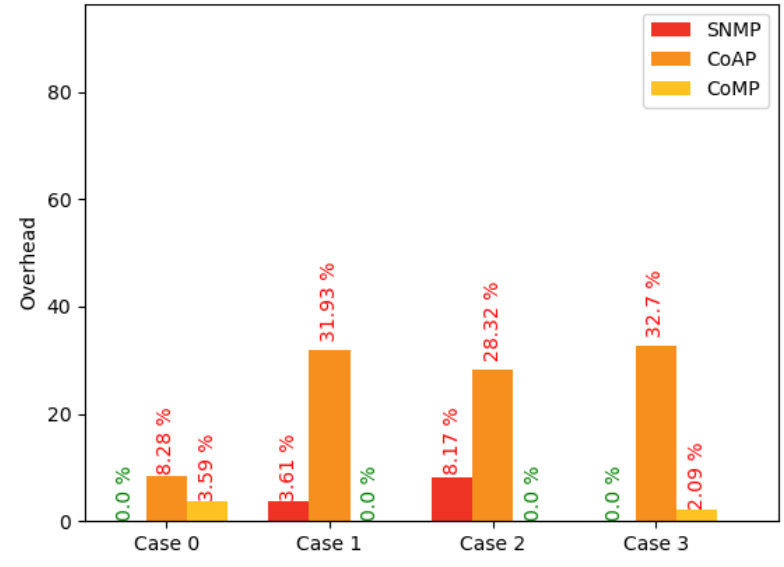

Fig. 15. Relative radio activity time (ms) for transmitting response packets with respect to the best performing protocol in FIT/IoT Lab test-bed experiments.

[8] W. Stallings, "SNMP and SNMPv2: the infrastructure for network management," IEEE Commun. Mag., vol. 36, no. 3, pp. 37-43, 1998.

[9] C. Bormann and P. Hoffman, "Concise Binary Object Representation (CBOR)," Internet Requests for Comments, RFC Editor, RFC 7049, 10 2013.

[10] F. Osterlind, A. Dunkels, J. Eriksson, N. Finne, and T. Voigt, "Crosslevel sensor network simulation with COOJA," Conference on Local Computer Networks, pp. 641-648, 2006.

[11] N. Accettura, L. A. Grieco, G. Boggia, and P. Camarda, "Performance analysis of the RPL Routing Protocol," IEEE International Conference on Mechatronics, Icm 2011 - Proceedings, pp. 5971 218, 767-772, 2011.

[12] R. Bhalerao, S. S. Subramanian, and J. Pasquale, "An analysis and improvement of congestion control in the coap internet-of-things protocol," 2016 13th Ieee Annual Consumer Communications and Networking Conference, Ccnc 2016, pp. 7444 906, 889-894, 2016.

[13] A. Elsts, X. Fafoutis, G. Oikonomou, R. Piechocki, and I. Craddock, "TSCH Networks for Health IoT: Design, Evaluation and Trials in the Wild," ACM Trans. Internet Things, vol. 1, no. 2, 2020.

[14] A. Elsts, X. Fafoutis, P. Woznowski, E. Tonkin, G. Oikonomou, R. Piechocki, and I. Craddock, "Enabling healthcare in smart homes: The sphere iot network infrastructure," IEEE Communications Magazine, vol. 56, no. 12, pp. 164-170, December 2018. [Online]. Available: https://doi.org/10.1109/MCOM.2017.1700791

[15] S. Duquennoy, B. Al Nahas, O. Landsiedel, and T. Watteyne, "Orchestra: Robust mesh networks through autonomously scheduled tsch," in Proceedings of the 13th ACM Conference on Embedded Networked Sensor Systems. New York, NY, USA: ACM, 2015, pp. 337-350.

[16] S. Duquennoy, J. Eriksson, and T. Voigt, "Five-nines reliable downward routing in RPL," CoRR, vol. abs/1710.02324, 2017. [Online]. Available: http://arxiv.org/abs/1710.02324

[17] C. Adjih, E. Baccelli, E. Fleury, G. Harter, N. Mitton, T. Noel, R. Pissard-Gibollet, F. Saint-Marcel, G. Schreiner, J. Vandaele, and T. Watteyne, "FIT IoT-LAB: A large scale open experimental IoT testbed," IEEE World Forum on Internet of Things, pp. 7389 098, 459464, 2015.

[18] I. Muhic and M. Hodzic, "Verification and validation of wireless sensor network energy consumption in internet of things," Icat 2017 - 26th International Conference on Information, Communication and Automation Technologies, Proceedings, vol. 2017-, pp. 1-6, 2017.

[19] M.-E.-A. Seddik, V. Toldov, L. Clavier, and N. Mitton, "From Outage Probability to ALOHA MAC Layer Performance Analysis in Distributed WSNs," IEEE Wireless Commun. and Networking Conf., 2018.

[20] X. Fafoutis, A. Elsts, A. Vafeas, G. Oikonomou, and R. Piechocki, "On Predicting the Battery Lifetime of IoT Devices: Experiences from the SPHERE Deployments," in Proceedings of the 7th International Workshop on Real-World Embedded Wireless Systems and Networks, 2018, p. 7-12.

[21] W. Stallings, "SNMPv3: A security enhancement for SNMP," IEEE Communications Surveys, vol. 1, no. 1, pp. 2-17, 1998. 